\title{
Prediction of Phase Composition and Nitrogen Concentration During the Nitriding Process in Low-Alloy Steel
}

\author{
Xiaohu Deng ${ }^{a}$, Dongying $\mathrm{Ju}^{b}$ \\ ${ }^{a}$ Tianjin Key Laboratory of high speed cutting and precision machining, Tianjin University \\ of Technology and Education - TUTE, Tianjin, 300222, China \\ ${ }^{b}$ Department of Mechanical Engineering, Saitama Institute of Technology - SIT, \\ Saitama, 369-0293, Japan
}

Received: February 26, 2015; November 26, 2016, 2015; Accepted: December 21, 2015

\begin{abstract}
A diffusion/transformation coupled model has been developed which combines finite difference (FD) model with a phenomenological model. The composition of the different iron-nitrogen( $\mathrm{Fe}-\mathrm{N})$ hardening phase can be regard as a function of nitriding time and nitrogen concentration. The diffusion model and transformation model are linked by the limiting nitrogen solubilities and the effective diffusion coefficients. The effect of alloy elements (Cr, Mo, Mn, V, Ni etc.) is considered by introducing an alloy coefficient for limiting nitrogen solubilities and diffusion coefficient. The diffusion/transformation model can predict nitrogen concentration, phase composition and hardness distribution. The model is employed to simulate the nitriding process of $\mathrm{SCr} 420 \mathrm{H}$ low-alloy steels. The simulated nitrogen concentration and hardness profiles are consistent with the measured ones. In addition, the predicted depth distributions of iron-nitrogen phase agree well with the available experimental results. Therefore, the comparison shows the reliability of the coupled model. It can be applied to improve the nitriding process parameters.
\end{abstract}

Keywords: Nitriding, Low-alloy steel, Fe-N phase composition, Nitrogen concentration, Modeling

\section{Introduction}

The nitriding process is one of the most important thermochemical treatment processes in metallurgy for the production of case-hardened surface layers in low-alloy steel. The diffusion of nitrogen into steel leads to the formation of a nitride layer, which comprises a thin outer compound layer (white layer) and thick inner diffusion layer. The compound layer consists of the epsilon phase $(\varepsilon)$ and the gamma prime phase $\left(\gamma^{\prime}\right)$. The diffusion layer is composed of interstitial solid solution of nitrogen dissolved in the ferrite lattice $(\alpha)^{1}$. The improvement of corrosion and wear properties can be attributed to the construct of compound layer. Therefore, it is beneficial to predict the nitrogen concentration profile and phase composition of the compound layer in the nitriding process.

To further understanding the nitriding process, numerical simulations have been employed to calculate the nitride layer growth and the nitrogen concentration profiles since $1990 \mathrm{~s}^{2-13}$. Sun and Bell ${ }^{2}$ developed a mathematical model to simulate the plasma nitriding process of low alloy steel. Their model considered the diffusion of nitrogen in ferrite and development of iron nitride layer on the surface simultaneously. Then, Goune et al., ${ }^{3}$ and Belmont et al., ${ }^{4}$ constructed a realistic diffusion-precipitation model to describe the volume diffusion of nitrogen in ferrite and the simultaneous precipitation of fine scale alloying element nitrides in the diffusion zone. Furthermore, Kamminga, Janssen ${ }^{8,9}$ presented a model for the calculation of nitrogen depth profiles in nitrided steel

*e-mail: dengxh@tute.edu.cn based on precipitation and trapping, and the calculations agreed well with experimental nitrogen depth profiles for nitrided Fe-Mn (1.62 wt.\% Mn) and Fe-V (0.55 wt.\% V) alloy. Keddam ${ }^{10}$ and Cavaliere et al., ${ }^{11}$ modeled the nitriding process by coupling the kinetics data of nitrogen in $\alpha, \gamma^{\prime}$ and $\varepsilon$ phases to the thermodynamic description of the iron-nitrogen (Fe-N) phase diagram respectively. Recently, Yang et al., ${ }^{12,13}$ presented a nitriding process model including the kinetics of compound layer growth and the determination of the nitrogen diffusivity in the diffusion zone. However, the prediction of $\mathrm{Fe}-\mathrm{N}$ phase composition and the hardness in the nitride layer have received less attention.

The main purpose of this paper is to simulate the nitrogen concentration, $(\mathrm{Fe}-\mathrm{N})$ phase composition and hardness in the nitriding process of low alloy steel. A diffusion/transformation coupled model is developed to predict the phase compositions at the thinner compound layer by considering simultaneous the thermodynamic data and kinetics theory in the nitriding process. The simulated results are compared with the experimental ones and theoretical descriptions.

\section{Model description}

The knowledge of both thermodynamic and kinetics data is required in modeling the nitriding process. A large number of thermodynamic and diffusion kinetics data can be found in ${ }^{14-20}$. Iron-nitrogen $(\mathrm{Fe}-\mathrm{N})$ binary phase diagrams are the base to understand the phase evolution during the nitriding process. In the past decades, the iron-nitrogen phase diagram 
and Fe-N system has been investigated extensively ${ }^{14,15}$. Moreover, Lakhtin ${ }^{16}$ reviewed the diffusion foundations of the nitriding process in carbon steel and alloy steel. Because the bonding energy in $\alpha$-phase is lower than that in the lattice of $\gamma^{\prime}$ and $\varepsilon$-phases, the coefficient of nitrogen diffusion in $\alpha$-phase should exceed the coefficients in the $\gamma^{\prime}$ and $\varepsilon$ by many times ${ }^{17}$. Furthermore, the phase composition of a compound zone and growth kinetics of a diffusion zone during the nitriding of steel was investigated quantitatively ${ }^{18,19}$. Recently, Somers ${ }^{20}$ reviewed the development of the compound layer during gaseous nitriding of Fe-based materials.

In the development of the nitriding model, in order to simplify model, the following assumptions are made:

Firstly, the diffusion process follows Fick's second law in a semi-infinite medium;

Secondly, the surface nitrogen concentration does not vary with the nitriding time;

Thirdly, a uniform temperature is assumed throughout the sample;

Lastly, local thermodynamic equilibrium is reached quickly at every point in the material.

\subsection{Nitrogen diffusion model}

The diffusion of nitrogen into ferrite phase is considered to obey Fick's second law ${ }^{21}$.

$\frac{\partial N}{\partial t}=D_{N}\left(\frac{\partial^{2} N}{\partial x^{2}}\right)$

where, Nis nitrogen concentration, $\chi$ is the depth, $t$ is nitriding time, $D_{n}$ denotes the effective diffusion coefficient of nitrogen.

The numerical method is applied to solve the diffusion equation. In the present model, an alternating-direction-implicit (ADI) finite-difference formulation is used to solve equation (1).

$$
\begin{aligned}
& C n_{i}^{t+1}=C n_{i}^{t}+F_{i}^{t} *\left(0.5 *\left(C n_{i-1}^{t}+C n_{i+1}^{t}+C n_{i-1}^{t+1}+C n_{i+1}^{t+1}\right)-\right. \\
& \left.C n_{i}^{t}-C n_{i}^{t+1}\right)
\end{aligned}
$$

where, $F_{i}^{t}=D_{i}^{t} * D_{t} / D X^{2}, D_{i}^{t}$ is the diffusion coefficient of location $i$ at time $t, D t$ is time step, $\mathrm{Cn}_{i}^{t}$ is the nitrogen concentration of location $i$ at time $t$.

The boundary conditions is the following

$$
\left.C(x, t)\right|_{x=0}=C_{N}^{S}
$$

where, $C_{N}^{S}$ is the surface nitrogen concentration.

\section{2. $\mathrm{Fe}-\mathrm{N}$ phase composition predicted model}

By employing the Fe-N phase diagram (Fig. 1 (a)) ${ }^{14,15}$, it is feasible to predict the different Fe-N phases according to the limit solubility values ${ }^{10,11}$. In Fig. 1 (b), it is presented the composition of the different $\mathrm{Fe}-\mathrm{N}$ phase as a function of nitriding time and nitrogen concentration. Based on the lever rule, a phenomenological model is developed to calculate the volume fraction of iron-nitrogen phase. The nitriding parameters are connected with the mechanisms of the
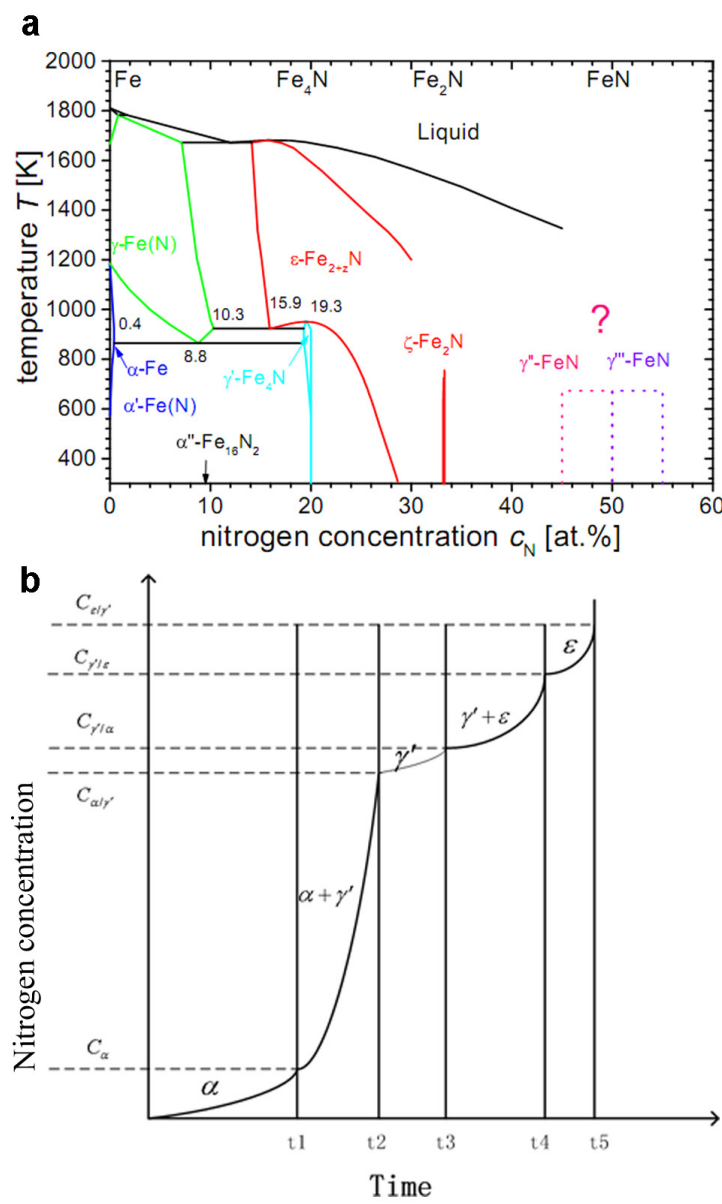

Fig. 1 a Fe-N phase diagram; b schematic illustration of Fe-N phases composition in the nitriding process.

nitriding process. The volume fraction of iron-nitrogen phases is defined as

$$
\begin{aligned}
\xi_{\gamma^{\prime}} & =\frac{C_{N}^{i, t}-C_{N}^{\alpha / \gamma^{\prime}}}{C_{N}^{\gamma^{\prime} / \alpha}-C_{N}^{\alpha / \gamma^{\prime}}}, \xi_{\varepsilon}=\xi_{\gamma^{\prime}} \cdot \frac{C_{N}^{i, t}-C_{N}^{\gamma^{\prime} / \varepsilon}}{C_{N}^{\varepsilon / \gamma^{\prime}}-C_{N}^{\gamma^{\prime} / \varepsilon}}, \\
\xi_{\alpha} & =1-\xi_{\gamma^{\prime}}-\xi_{\varepsilon}
\end{aligned}
$$

where, $C_{N}^{i, t}$ is nitrogen concentration, $C_{N}^{\alpha / \gamma^{\prime}}, C_{N}^{\gamma^{\prime / \alpha}}, C_{N}^{\gamma^{\prime} / \varepsilon}, C_{N}^{\varepsilon / \gamma^{\prime}}$ are the limiting nitrogen solubilities in $\mathrm{Fe}-\mathrm{N}$ phase, as a function of temperature can be calculated by ${ }^{10,11,16}$

$$
\begin{aligned}
C_{N}^{\alpha / \gamma^{\prime}} & =\exp \left(-\frac{4575}{T}+3\right) \cdot 10^{\gamma_{A E}} \\
C_{N}^{\gamma^{\prime} / \alpha} & =\frac{25.08}{4.25+10^{-\left(\frac{2341.67}{T}-1.925\right)}} \\
C_{N}^{\gamma^{\prime} / \varepsilon} & =\frac{25.08}{4.25+10^{-\left(\frac{3476.67}{T}-2.455\right)}} \\
C_{N}^{\varepsilon / \gamma^{\prime}} & =\left(3.01+\left(1.79 \cdot 10^{-2} \mathrm{~T}\right)-\left(1.54 \cdot 10^{-5} \mathrm{~T}^{2}\right)\right) \cdot 10^{\gamma_{A E}}
\end{aligned}
$$


where, $\gamma_{A E}$ is the activity coefficient of nitrogen, which can be calculated by 22

$$
\begin{aligned}
& \gamma_{A E}=-\left(0.054 \times C m o-0.127 \times C c r^{0.9}+0.047 \times\right. \\
& \left.C m n+0.083 \times C w+0.027 \times C n i-0.011 \times C a l^{0.35}\right)
\end{aligned}
$$

where, $C_{i}$ is the wt. $\%$ of $i$ element.

\subsection{Hardness regression model}

The hardness could be calculated by the experimental regression equation ${ }^{5,7}$

$$
H V=H V_{s t}+\sum_{x=1}^{n 1} a_{x} C_{x}+\sum_{y=1}^{n 2} b_{y} \xi_{c y}+\sum_{z=1}^{n 3} c_{z} \xi_{n z}
$$

where, $H V$ is the Vickers Hardness, $H V_{s t}$ is the Vickers Hardness of the substrate, $C_{x}$ is the chemical compositions, $\xi_{c y}$ is the iron-carbon phase compositions, $\xi_{n z}$ is the Fe-N phase compositions, and $a x(x=S i, M n, N i$ and $C r)$, $b y(\mathrm{y}=M, B, A$ and $F), c Z\left(\mathrm{z}=\varepsilon, \gamma^{\prime}\right)$ are the corresponding weighting coefficients, which obtained by literature ${ }^{23-25}$ and experimental data. The values of coefficients are listed in Table 1.

\subsection{Diffusion/Transformation coupled program}

The diffusion equation and transformation equation were linked by the effective diffusional coefficients, which could be given by the mixture law ${ }^{5,6}$

$$
D_{N}=\sum D_{N}^{i} \xi_{i}
$$

where, for each single phase, $i=1(\varepsilon), 2\left(\gamma^{\prime}\right), 3(\alpha), D_{N}^{i}$ is diffusivity in various $\mathrm{Fe}-\mathrm{N}$ phases, $\xi_{i}$ is volume fraction of various Fe-N phase. The diffusivity of nitrogen in different $\mathrm{Fe}-\mathrm{N}$ phase could be approximately expressed in an Arrhenius form ${ }^{10,11,16,17}$

$$
\begin{aligned}
& D_{N}^{\alpha}=\eta_{a l}^{\alpha} 6.6 \times 10^{-7} \exp (-77820 / R T) \\
& D_{N}^{\gamma^{\prime}}=\eta_{a l}^{\gamma^{\prime}} 1.675 \times 10^{-9} \exp (-64000 / R T) \\
& D_{N}^{\varepsilon}=\eta_{a l}^{\varepsilon} 2.1 \times 10^{-8} \exp (-93517 / R T)
\end{aligned}
$$

where, $\eta_{a l}^{\alpha}, \eta_{a l}^{\gamma^{\prime}}$ and $\eta_{a l}^{\varepsilon}$ are the alloy coefficients, which can be determined by the formula ${ }^{16}$

$$
\eta_{a l}=\exp \left[\sum B_{i}(\% A E)\right]^{n_{i} / T}
$$

where $(\% A E)$ is the concentration of the alloying element in the steel, $B_{i}$ and $n_{i}$, are coefficients determined on the basis of experimental data on the thickness of the nitrided layer in binary alloys. The flowchart of coupled program is shown in Fig. 2. In the present program, the diffusion and transformation are calculated respectively. On the one hand, the effective diffusion coefficients are changed with the iron-nitrogen phase fraction by equation (8); On the other hand, the iron-nitrogen fraction can be recalculated by equation (4).

\section{Results and discussions}

In order to validate model, the diffusion/transformation coupled model is applied to predict the nitriding process of $\mathrm{SCr} 420 \mathrm{H}$ steel. The chemical compositions of specimens are listed in Table 2. The nitriding process parameters are shown in Fig. 3. Hardness measurements on cross sections and the surfaces of nitrided specimens were carried out with a Vickers hardness tester. For the determination of nitrogen contents, Electron Probe X-ray Microanalysis (EPMA) was performed on cross-sections of the specimens.

Fig. 4 depicts the evolutions of the nitrogen concentration profiles versus depth for nitriding of $\mathrm{SCr} 420 \mathrm{H}$ steel. The profiles show the comparison between the simulated and experimental data. Although there are detailed discrepancies, it can be seen that good agreement is achieved between the numerical results and Wavelength Dispersive (WDS) measured results. It appears that the nitrogen concentration of diffusion layer is underestimated. In the present model, the distributions of nitrogen concentration depend on the effective diffusional coefficients, which are relevant to the concentration of the alloying element (equation (9) and (10)). Therefore, the assumption that the chemical elements are uniform distribution may result in inaccurate diffusional coefficient and nitrogen concentration distribution. It is found that the nitrogen concentration gradient adjacent to the surface is very steep, while away from the surface is flat. It is indicated the diffusivity of nitrogen greatly change in different depth of sample.

In Fig. 5, the volume fractions of the different hardening phase are shown in $\mathrm{SCr} 420 \mathrm{H}$ steel. It can be seen that the nitride layer is composed of the thinner compound layer $\left(\gamma^{\prime}+\varepsilon\right)$ and inner diffusion layer $\left(\alpha+\gamma^{\prime}\right)$. The simulated depth distributions of $\gamma^{\prime}$ and $\varepsilon-F e_{2-3} N$ phases are reasonable consistent with the X-ray diffraction (XRD) phase analysis results ${ }^{19}$ and optical micrograph. The formation of $\gamma^{\prime}$ phase subzone in the inner part of the compound zone may

Table 1 - The values of coefficients for hardness calculation

\begin{tabular}{cccccccccccc}
\hline Coefficients & $\boldsymbol{H} \boldsymbol{V}_{\boldsymbol{s t}}$ & $\boldsymbol{a}_{\boldsymbol{s i}}$ & $\boldsymbol{a}_{\boldsymbol{M} \boldsymbol{n}}$ & $\boldsymbol{a}_{\boldsymbol{N} \boldsymbol{i}}$ & $\boldsymbol{a}_{\boldsymbol{C r}}$ & $\boldsymbol{b}_{\boldsymbol{M}}$ & $\boldsymbol{b}_{\boldsymbol{F}}$ & $\boldsymbol{b}_{\boldsymbol{B}}$ & $\boldsymbol{b}_{\boldsymbol{A}}$ & $\boldsymbol{C}_{\boldsymbol{\varepsilon}}$ & $\boldsymbol{C}_{\boldsymbol{\gamma}^{\prime}}$ \\
\hline Values (HV) & 196 & 27 & 11 & 8 & 16 & 745 & 212 & 632 & 201 & 983 & 886 \\
\hline
\end{tabular}

Table 2 - Chemical composition of SCr420H steel

\begin{tabular}{cccccccccc}
\hline Chemical elements & Fe & $\mathbf{C}$ & $\mathbf{S i}$ & $\mathbf{M n}$ & $\mathbf{C r}$ & $\mathbf{P}$ & $\mathbf{S}$ & $\mathbf{N}$ & Mo \\
\hline Wt.\% & 94 & 0.21 & 0.19 & 0.80 & 1.15 & 0.0017 & 0.0015 & 0.00 & 0.02 \\
\hline
\end{tabular}


upset the balance of the nitrogen concentration between the diffusion zone and the compound zone, and may as a consequence have an influence upon the kinetics of the diffusion zone growth. The volume fraction of Fe-N phases is corresponding to the nitrogen concentration (Fig. 4). At the outer section, the nitrogen concentration is relatively high. At the inner section, nitrogen concentration is decreased rapidly to a lower value.

The hardness profiles after nitriding are calculated by experimental regression equation (4) and shown in Fig. 6 for $\mathrm{SCr} 420 \mathrm{H}$ steel. The calculated hardness data result in good agreement with the measured ones. However, there are discrepancies between calculations and observations for the maximum hardness. The problem probably lies in the estimated hardness value of alloy nitride in regression equation, which can influence hardness in diffusion layers significantly. As shown as in Fig. 4-Fig. 6, it can be concluded that the coupled model can be employed to predict the hardening phase composition in the nitriding process quantitatively.

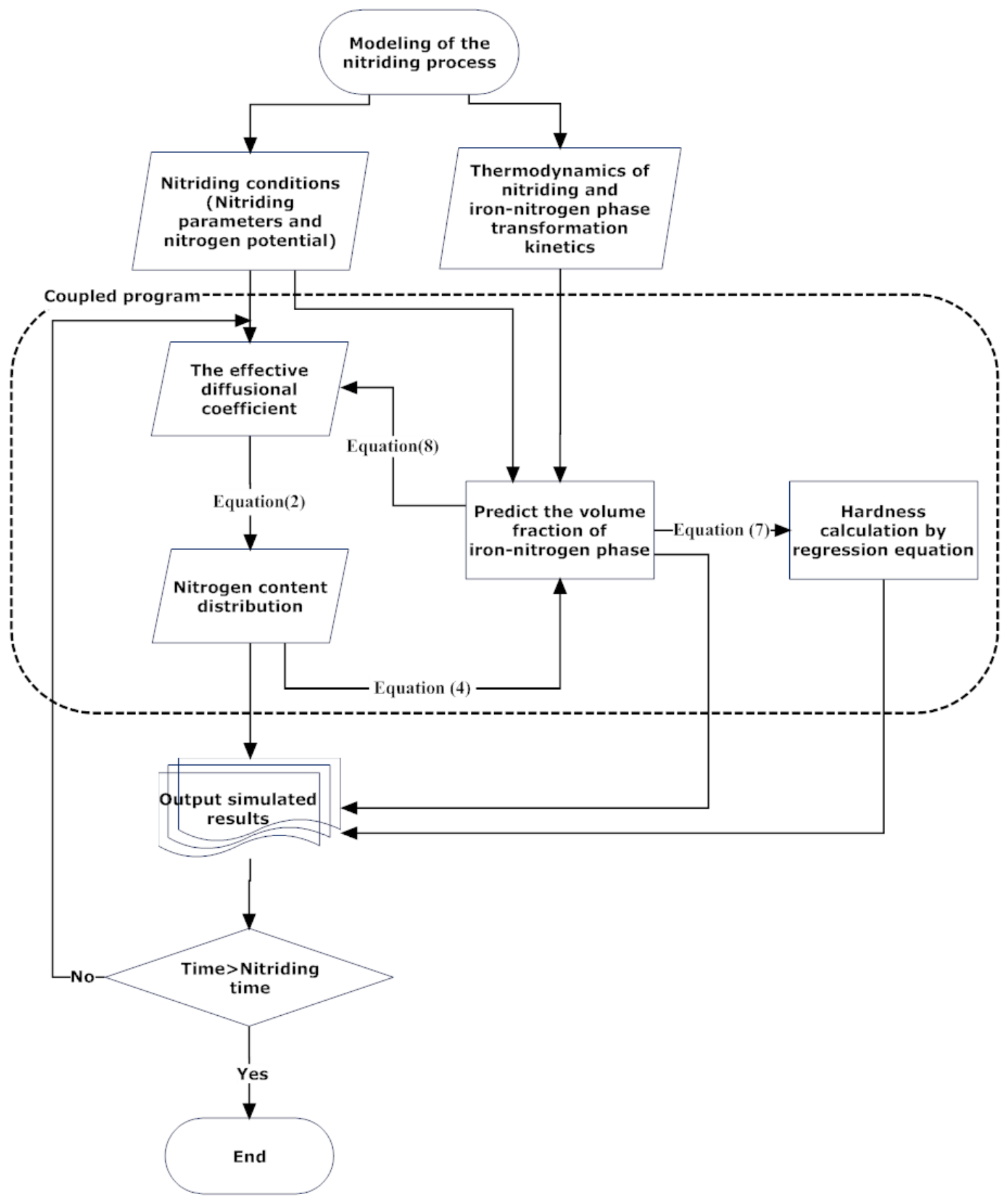

Fig. 2 The flowchart of the diffusion/transformation coupled program. 


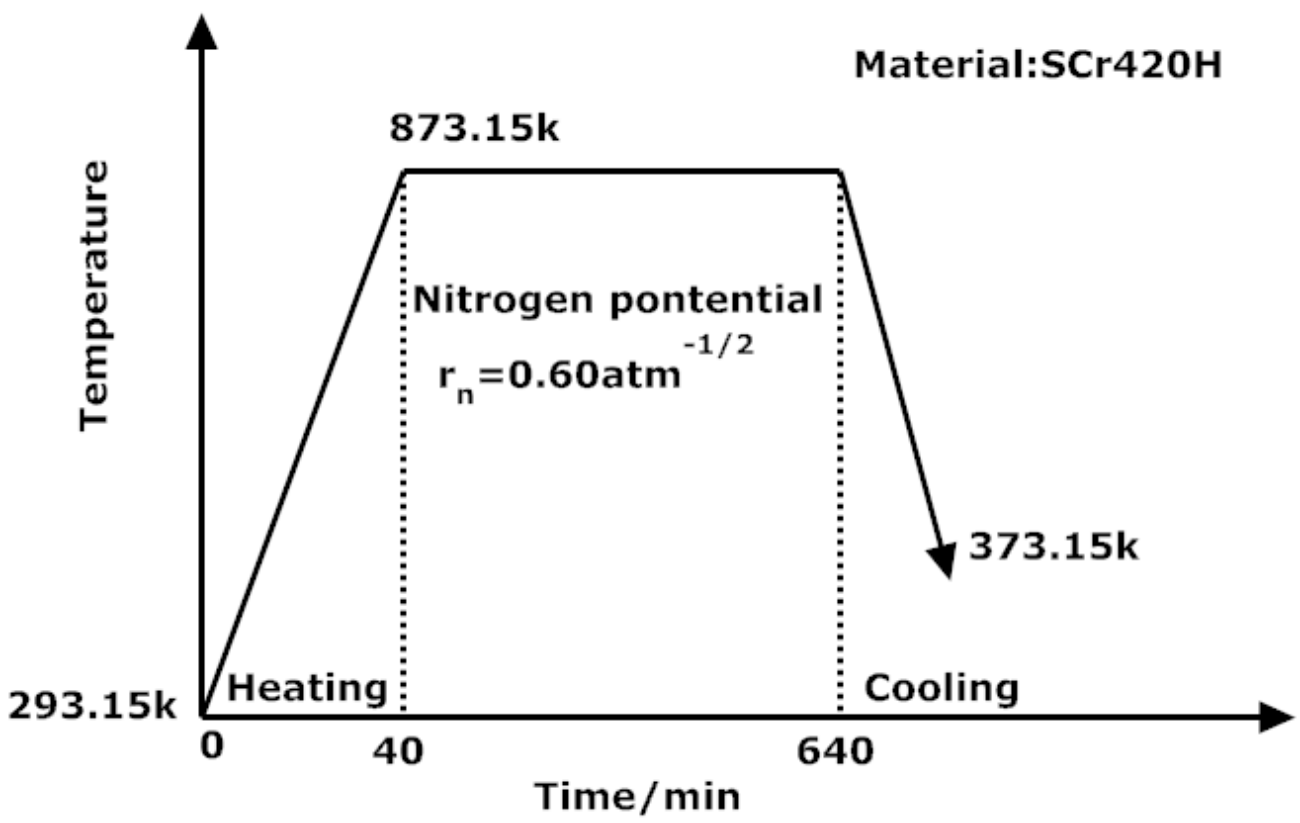

Fig. 3 A schematic illustration for nitriding process in $\mathrm{SCr} 420 \mathrm{H}$ steel.

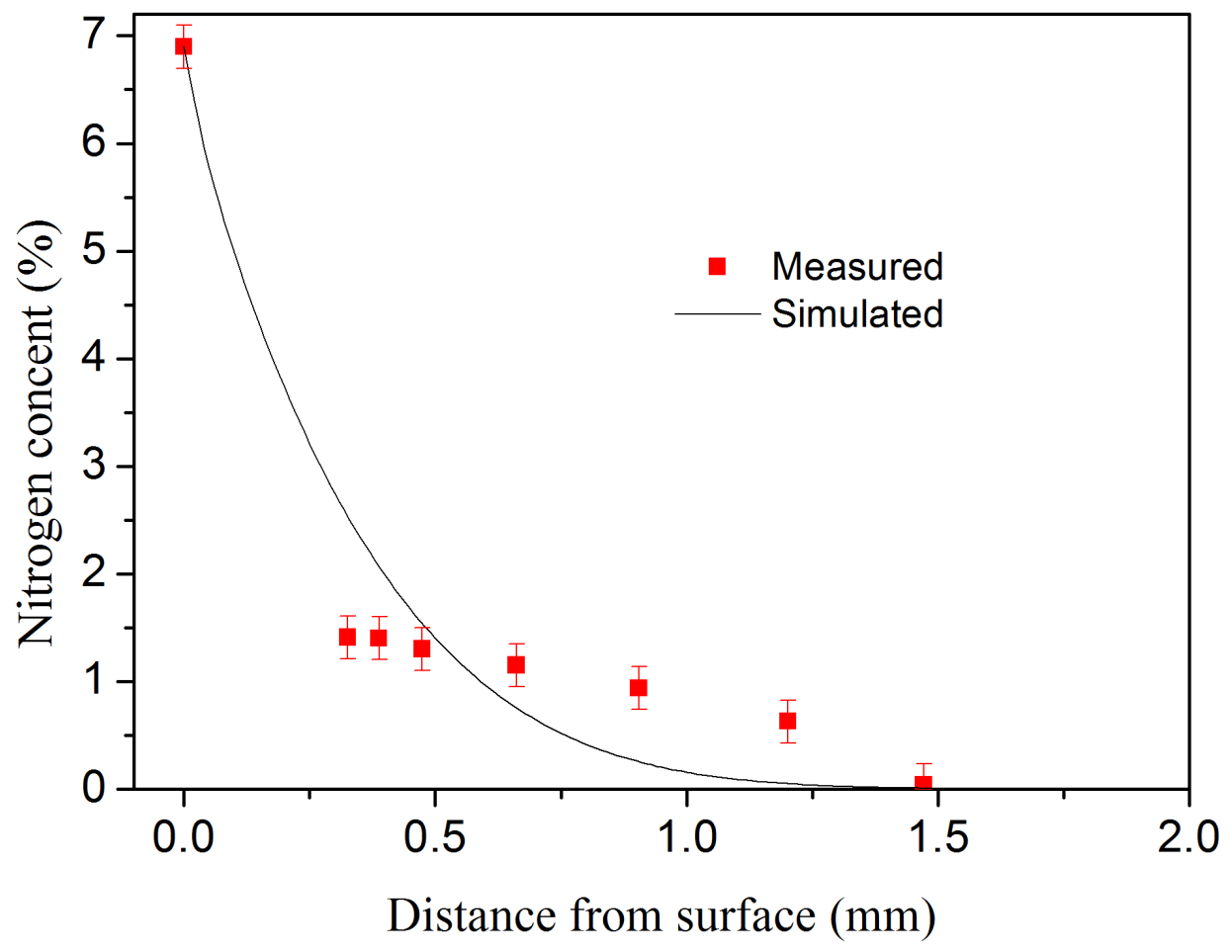




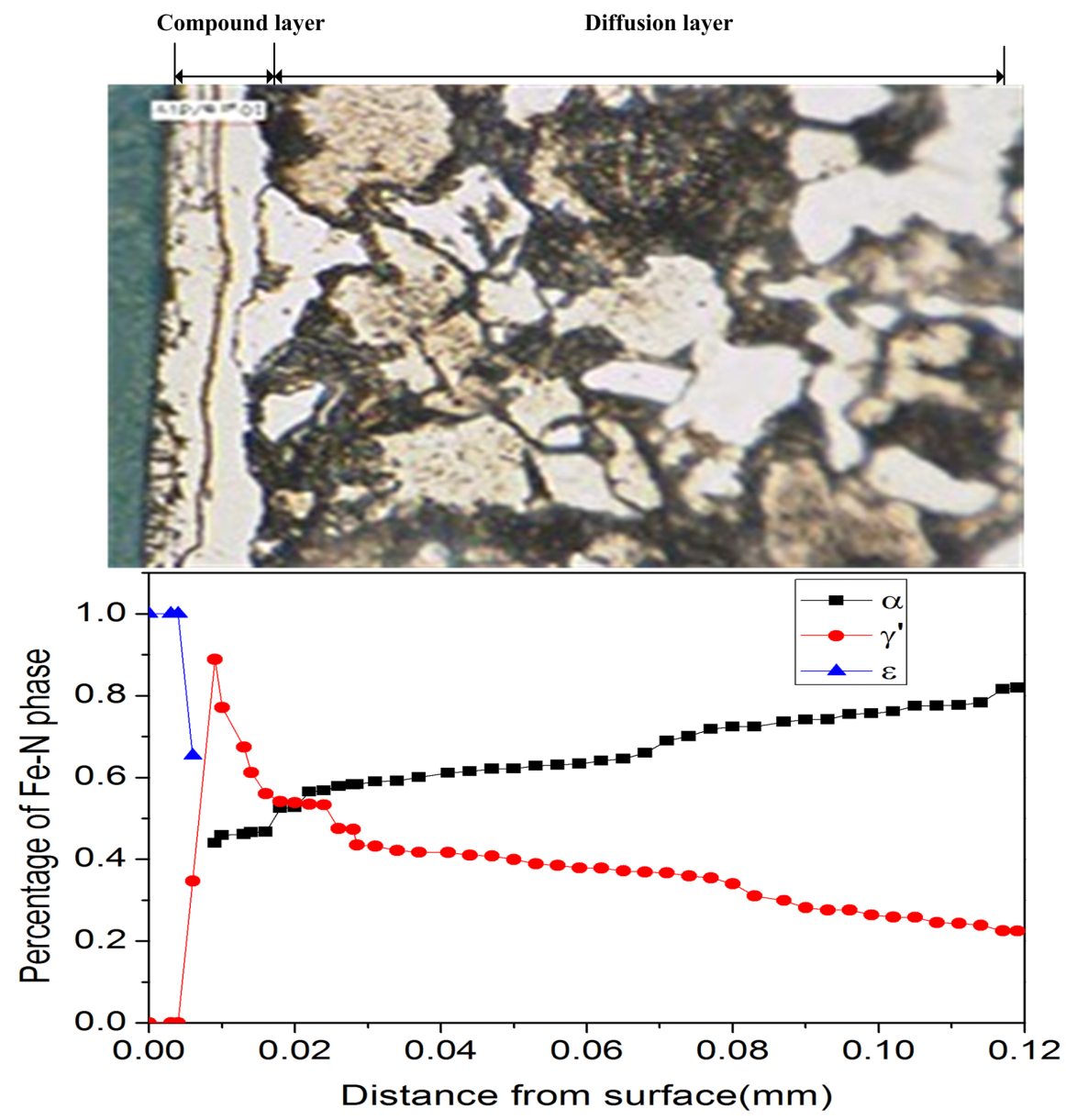

Fig. 5 Volume fraction of the Fe-N phase in $\mathrm{SCr} 420 \mathrm{H}$ steel.

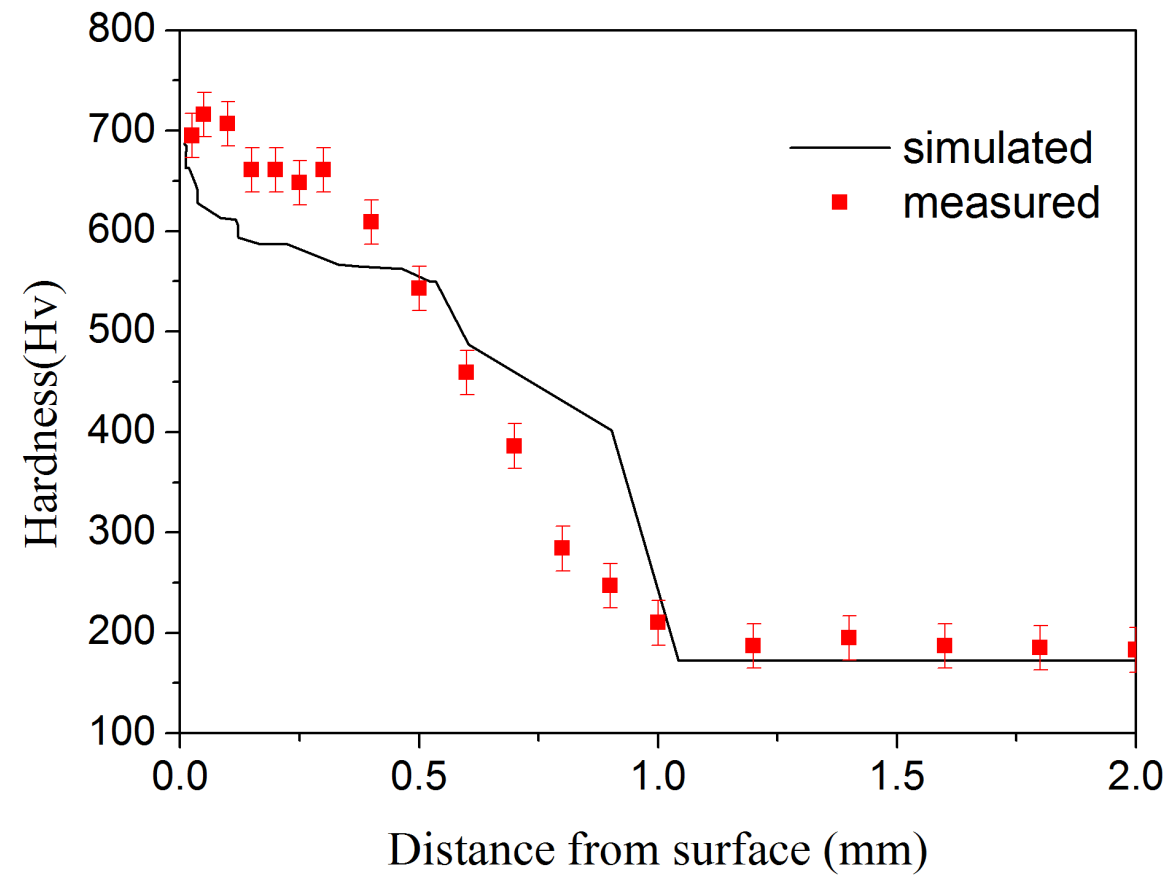

Fig. 6 Hardness distributions in $\mathrm{SCr} 420 \mathrm{H}$ steel 


\section{Conclusions}

In this paper, a diffusion/transformation coupled model has been developed to simulate nitrogen concentration distributions, iron-nitrogen phase formation and hardness by combining nitrogen diffusion and $\mathrm{Fe}-\mathrm{N}$ phase composition calculation. The model is implemented to simulate nitriding process of $\mathrm{SCr} 420 \mathrm{H}$ steel. The simulated results indicate the nitrogen concentration profiles, volume fraction of $\mathrm{Fe}-\mathrm{N}$ phases and hardness distributions can be predicted quantitatively by this model. Moreover, the simulated results are in reasonable agreement with the experimentally measured ones and theoretical analysis. It shows the

\section{References}

1. Pye D. Practical nitriding and ferritic nitrocarburizing. Materials Park, OH: ASM International; 2003.

2. Sun Y, Bell T. A numerical model of plasma nitriding of low alloy steels. Materials Science \& Engineering A. 1997;224(1): 33-47.doi:10.1016/S0921-5093(96)10561-X

3. Goune M, Belmonte T, Fiorani JM, Chomer S, Michel H. Modelling of diffusion-precipitation in nitrided alloyed iron. Thin Solid Films. 2000;377-378:543-549.doi:10.1016/S00406090(00)01305-5

4. Belmonte T, Gouné M, Michel H. Numerical modeling of interstitial diffusion in binary systems: Application to iron nitriding. Materials Science \& Engineering A. 2001;302(2): 246-57.

5. Liu CC, Ju DY, Inoue T. A numerical modeling of metallothermo-mechanical behavior in both carburized and carbonitrided quenching processes. ISIJ International. 2002;42(10): 11251134.doi:10.1016/S0924-0136(03)00378-9

6. Ju DY, Liu CC, Inoue T. Numerical modeling and simulation of carburized and nitrided quenching process. Journal of Materials Processing Technology. 2003;143-144:880-885.doi:10.1016/ S0924-0136(03)00378-9

7. Krukovich MG. Simulation of the nitriding process. Metal Science and Heat Treatment. 2004;46(1):25-31.

8. Kamminga JD, Janssen GC. Calculation of nitrogen depth profiles in nitrided Fe-Mn and Fe-V. Surface \& Coatings Technology. 2006;200(20):909-912.

9. Kamminga JD, Janssen GC. Calculation of nitrogen depth profiles in nitrided multi-component ferritic steel. Surface \& Coatings Technology. 2006; 200(20-21): 5896-5901.doi:10.1016/j. surfcoat.2005.09.002

10. Keddam M. Surface modification of the pure iron by the pulse plasma nitriding: Application of a kinetic model. Materials Science \& Engineering A. 2007;462(1): 169-173. doi:10.1016/j. msea.2006.02.459

11. Cavaliere P, Zavarise G, Perillo M. Modeling of carburizing and nitriding processes. Computational Materials Science. 2009;46(1): 26-35.

12. Yang M, Yao B, Sohn YH, Sisson Jr RD. Simulation of the ferritic nitriding proces. International Heat Treatment and Surface Engineering. 2011;5(3):122-126. DOI: http://dx.doi. org/10.1179/174951411X12956208225186 possibility of utilizing the model to improve the nitriding process parameters.

\section{Acknowledgements}

This research receives ongoing support from the High-tech Research Center and Open Research Center at the Saitama Institute of Technology, and sponsored by Natural Science Foundation of Tianjin (No. 13JCYBJC38900), National Natural Science Foundation of China (Project 51301121), Scientific Research Starting Foundation of Tianjin University of Technology and Education (No. KYQD12008) and Innovation Team Training Plan of Tianjin Universities and colleges (Grant No. TD12-5043).

13. Yang M, Zimmerman C, Donahue D, Sisson Jr RD. Modeling the gas nitriding process of low alloy steels. Journal of Materials Engineering and Performance. 2013;22(7):1892-1898.

14. Wriedt HA, Gokcen NA, Nafziger RH. The Fe-N (Iron-Nitrogen) system. Bulletin of Alloy Phase Diagrams. 1987;8:355-377.

15. Voorthuysen EH, Boerma DO, Chechnin NC. Low-temperature extension of the lehrer diagram and the iron-nitrogen phase diagram. Metallurgical and Materials Transactions A. 2002;33(8):2593-2598.

16. Lakhtin YU. Diffusion foundations of the nitriding process. Metal Science and Heat Treatment. 1995;37(7):276-279.

17. Somers MA, Mittemeijer EJ. Layer-growth kinetics on gaseous nitriding of pure iron: Evaluation of diffusion coefficients for nitrogen in iron nitrides. Metallurgical and Materials Transactions A. 1995;26(1):57-74.

18. Hernandez M, Staia MH, Puchi-Cabrera ES. Evaluation of microstructure and mechanical properties of nitrided steels. Surface \& Coatings Technology. 2008;202(10):1935-1943. doi:10.1016/j.surfcoat.2007.08.018

19. Ratajski J. Relation between phase composition of compound zone and growth kinetics of diffusion zone during nitriding of steel. Surface \& Coatings Technology. 2009; 203(16):23002306.DOI: 10.1016/j.surfcoat.2009.02.021

20. Somers MA. Development of compound layer during nitriding and nitrocarburising; current understanding and future challenges. International Heat Tretament and Surface Engineering. 2011;5(1):7-16.

21. Crank J. The mathematics of diffusion. Oxford: Clarendon Press; 1956.

22. Lakhtin YU, Kogan YA, Bulgach AA. Effect of alloying elements on the thermodynamic activity and solubility of nitrogen in phases of a nitrided case. Metal Science and Heat Treatment. 1982;24(4):15-18.

23. Yakhnina VD, Nikitin VV. Development of the hardness of the nitrided case. Metal Science and Heat Treatment. 1975;17(12):125-128.

24. Lakhtin YU, Kogan YA, Sharlat ES. Nitriding of low- and medium-carbon steels surface-alloyed with nitride-forming elements. Soviet Materials Science. 1976; 13(5):514-517.

25. Glushchenko VN, Goryushin VV, Kondrashova GA, Duka ME. Effect of alloying on the structure and properties of the nitrided case on medium-carbon steels. Metal Science and Heat Treatment. 1980;22(9):638-643. 